\title{
Adding-weight One- rank Local Predication Model of Busy Traffic based on Correlation
}

\author{
Zhimei Duan $^{1, a^{*}}$, Xiaojin Yuan ${ }^{2, b}$ and Yan Xiong ${ }^{3, c}$ \\ ${ }^{1}$ The Engineering College of Honghe University, Mengzi, 661199, China \\ ${ }^{2}$ Comprehensive Test Center, Honghe Quality and Technical Supervision, Mengzi, 661199, China \\ ${ }^{3}$ The Engineering College of Honghe University, Mengzi, 661199, China \\ a704424481@qq.com, b494182567@qq.com, ccjt@163.com
}

Keywords: Correlation; Parameter identification; Adding-weight one-rank local prediction model; Busy traffic; Prediction

\begin{abstract}
In order to improve accuracy of adding-weight one-rank local prediction, the paper proposed the correlation function to measure the correlation between different phase points, which is used to determine reference neighborhood of prediction center point, and made the values of expressing correlation to act on model by means of adding-weight, derived the identification algorithm of model parameters. Applied prediction model to prediction of busy traffic; the results show that the model effectively improves prediction accuracy of busy traffic, verifying the relevant function to measure the effectiveness of the phase correlation between different phase points.
\end{abstract}

\section{Introduction}

Forecasting of busy traffic, can undertake network security for network operation and provide decision-making support, it is an important basis for mobile operators to design network. Whether predicting result is accurate or not, it will affect communication quality. Busy traffic is a non-linear time series affected by multiple factors. The traditional forecast is season, ARMA model and regression, etc., but they mainly for smooth traffic forecast. It is difficult to satisfy the prediction of busy traffic. Some scholars put forward BP neural network [1], support vector machine [2], and echo state network [3], etc. these methods can deal with the non-linear relationship between traffic and its impact factors; they have more advantages than the traditional prediction.

Chaos is a new form of non-linear systems, its prediction has global and local [4], [5] using adding-weight one-rank local model to forecast short-term load, but model base on Euclidean distance to measure correlation between phase points, when embedding dimension is higher, Euclidean distance only reflect the distance of phase space, it can't fully reflect the correlation [6,7] of phase. Some scholars put forward to describe correlation of phase trajectory similar degree instead of Euclidean distance to measure correlation, which improves precision of short-term load. However the research of [8] and [9] shows that measured by phase Angle between the phase trajectories of similarity degree has better prediction effect than the correlation.

In fact, correlation between phase points, not only shorten space distance between phase points but also hope that the angle between phase trajectories of evolution direction is small to ensure the similarity of track changes. So, the paper considers the both influence on correlation, proposed discriminate correlation between phase points on the basis of the correlation function, deduced the new algorithm, finally model will be used in forecast of busy traffic to verify prediction effect.

\section{Analysis of Adding-weight One-rank Local Model}

Set observation data is $\left\{x_{1}, x_{2}, \ldots, x_{N}\right\}, N$ is the length of data, if the appropriate time delay $\tau$ and the best embedding dimension $m$ is selected, it can turn into a new set of vector series.

$$
X_{i}=\left[x_{i}, x_{i+}, \ldots, x_{i+(m-1)}\right]^{T}, i=1,2, \ldots, M, M=N-(m-1) \tau
$$

The reconstruction of phase space is made of the observed data, $\tau$ and $m$. According to the law of Taken [6], the phase space can be recovery in the sense of topological equivalence dynamics 
characteristic of time series, namely a trajectory under the high dimensional space.

If predicting point is $x_{N+1}$, the $m$ th component of phase point $X_{M+1}, X_{M}$ is predicting center. calculate the Euclidean distance with $X_{M}$ and the rest of the phase point in phase space and choose $q$ neighboring points which is close to $X_{M k}(k=1,2,3, \ldots, q)$ as its reference neighborhood, then adding-weighted one-rank local prediction model fitting $k$ reference neighborhood points.

$X_{M_{k}+1}=A+B X_{M_{k}}$

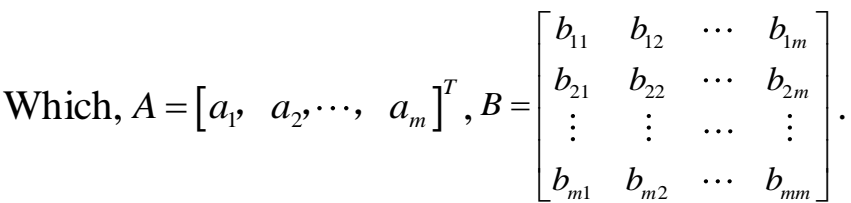

In type (2), the $m$ th can be predicted:

$$
x_{M_{k}+1}^{m}=a_{m}+\sum_{j=1}^{m} b_{m j} x_{M_{k}}^{j}
$$

Set $X_{M k}$ normalized weight is $P_{k}$, the adding-weighted least square method as follows.

$$
\sum_{k=1}^{q} P_{k}\left(x_{M_{k}+1}^{m}-a_{m}-\sum_{j=1}^{m} b_{m j} x_{M_{k}}^{j}\right)^{2}=\min
$$

Regard (4) as a function about $a_{m}, b_{m j}(j=1,2, \cdots, m)$, calculate the partial derivatives of $a_{m}, b_{m j}$, prediction model of fitting parameters $a_{m}, b_{m j}$, obtain prediction model of predicting point $x_{N+1}$ :

$$
\hat{x}_{N+1}=\hat{x}_{M+1}^{m}=a_{m}+b_{m 1} x_{M}^{1}+b_{m 2} x_{M}^{2}+\cdots b_{m m} x_{M}^{m}=a_{m}+\sum_{j=1}^{m} b_{m j} x_{M}^{j}
$$

Predicting principle is shown in Fig. 1.

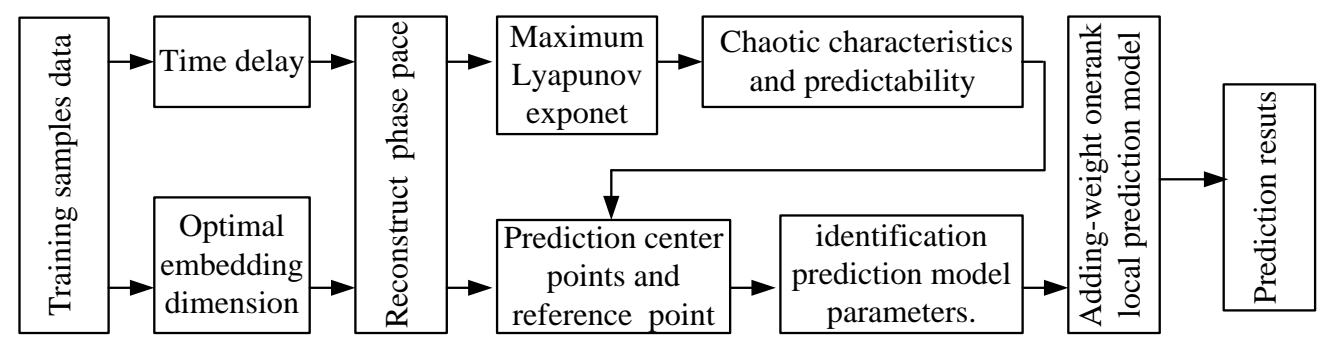

Figure 1. Prediction principle of adding-weight one-rank local

\section{Adding-weighted One-rank Local Prediction Model Based on Relevance}

Correlation Functions of Measured Phase Point. According to the reconstructed phase space, set Euclidean distance between point $X_{i}(i \neq M)$ and predicting center point $X_{M}$ : Set $d_{\min }$ as the minimum value of $\mathrm{d}_{i}$, and used $\mathrm{y}_{i}$ in describing phase point $X_{i}$ to forecast the influence degree of center of $X_{M}$ :

$$
\begin{aligned}
& d_{i}=\left[\sum_{j=1}^{m}\left(X_{M}^{j}-X_{i}^{j}\right)^{2}\right]^{\frac{1}{2}} \\
& y_{i}=\frac{\exp \left\{-\left(d_{i}-d_{\min }\right)\right\}}{\sum_{i=1}^{M-1} \exp \left\{-\left(d_{i}-d_{\min }\right)\right\}}
\end{aligned}
$$

Which, $\mathrm{d}_{\mathrm{i}} \in[0, \infty), \mathrm{y}_{i} \in(0,1)$. If $\mathrm{d}_{i} \rightarrow 0, X_{i}$ and $X_{M}$. is closer, then $\mathrm{y}_{i} \rightarrow 1$, the greater influence of $\mathrm{X}_{i}$ to predict points.

Set $X_{i}(i \neq \mathrm{M})$ and Angle cosine of $X_{M}$ is as follows.

$$
\cos \left(X_{M}, X_{i}\right)=\frac{X_{M} \cdot X_{i}}{\left|X_{M}\right| \times\left|X_{i}\right|}=\frac{\sum_{j=1}^{m} X_{M}^{j} \times X_{i}^{j}}{\sqrt{\sum_{j=1}^{m}\left(X_{M}^{j}\right)^{2}} \times \sqrt{\sum_{j=1}^{m}\left(X_{i}^{j}\right)^{2}}}
$$


Using $z_{i}$ to show the shape similarity in trajectory with phase point $X_{i}$ and $X_{M}$ :

$z_{i}=\frac{\cos \left(X_{M}, X_{i}\right)+1}{2}$

Which $\cos \left(X_{M}, X_{i}\right) \in[-1,1], z_{i} \in[0,1]$. If $\cos \left(X_{M}, X i\right) \rightarrow 1$, then $\mathrm{z}_{i} \rightarrow 1$, the phase trajectory is similar, the higher the precision is.

Set a measure of the correlation of $X_{i}$ and $X_{M}$ correlation function for $\mathrm{c}_{i}, \mathrm{c}_{i}=\mathrm{y}_{\mathrm{i}} \mathrm{z}_{i}$, and $c_{i} \in[0,1], c_{i}$ considered influence of Euclidean distance and Angle cosine on the correlation between $X_{M}$ and $X_{i}$, if $c_{i} \rightarrow 1$, then the stronger impact on prediction results in correlation.

Parameter Identification Adding-weight One-rank Local Forecast Model. Selected the largest phase points $\mathrm{X}_{M_{k}}(k=1,2 \ldots \mathrm{q})$ of the $q$ value of $c_{i}$ as a neighborhood reference. Set $c_{\max }$ for maximum value of $c_{i}, c_{k}$ is correlation of the $k$ adjacent points, and the $k$ reference neighborhood point's weight is as follows.

$$
P_{k}=\frac{\exp \left(c_{k}-c_{\max }\right)}{\sum_{k=1}^{q} \exp \left(c_{k}-c_{\max }\right)}
$$

Type (10) into type (4), calculate the partial derivatives of $a, b m j(j=1,2,3, m)$ respectively, then obtain expansion equations of $a, b_{m j}$ as follows.

$$
\begin{aligned}
& \sum_{k=1}^{q}\left(P_{k} x_{M_{k}+1}^{m}\right)=a_{m}+b_{m 1} \sum_{k=1}^{q}\left(P_{k} x_{M_{k}}^{1}\right)+b_{m 2} \sum_{k=1}^{q}\left(P_{k} x_{M_{k}}^{2}\right)+\cdots b_{m m} \sum_{k=1}^{q}\left(P_{k} x_{M_{k}}^{m}\right) \\
& \sum_{k=1}^{q}\left(P_{k} x_{M_{k}+1}^{m} x_{M_{k}}^{1}\right)=a_{m} \sum_{k=1}^{q}\left(P_{k} x_{M_{k}}^{1}\right)+b_{m 1} \sum_{k=1}^{q}\left[P_{k}\left(x_{M_{k}}^{1}\right)^{2}\right]+b_{m 2} \sum_{k=1}^{q}\left(P_{k} x_{M_{k}}^{1} x_{M_{k}}^{2}\right)+\cdots b_{m m} \sum_{k=1}^{q}\left(P_{k} x_{M_{k}}^{m} x_{M_{k}}^{1}\right) \\
& \vdots \\
& \sum_{k=1}^{q}\left(P_{k} x_{M_{k}+1}^{m} x_{M_{k}}^{m}\right)=a_{m} \sum_{k=1}^{q}\left(P_{k} x_{M_{k}}^{m}\right)+b_{m 1} \sum_{k=1}^{q}\left[P_{k} x_{M_{k}}^{1} x_{M_{k}}^{m}\right]+b_{m 2} \sum_{k=1}^{q}\left(P_{k} x_{M_{k}}^{2} x_{M_{k}}^{m}\right)+\cdots b_{m m} \sum_{k=1}^{q}\left[P_{k}\left(x_{M_{k}}^{m}\right)^{2}\right]
\end{aligned}
$$

Matrix form of type (11) is as follows.

$F=G H$

$$
\text { Which, } F=\left[\begin{array}{c}
\sum_{k=1}^{q}\left(P_{k} x_{M_{k}+1}^{m}\right) \\
\sum_{k=1}^{q}\left(P_{k} x_{M_{k}+1}^{m} x_{M_{k}}^{1}\right) \\
\vdots \\
\sum_{k=1}^{q}\left(P_{k} x_{M_{k}+1}^{m} x_{M_{k}}^{m}\right)
\end{array}\right], G=\left[\begin{array}{cccc}
1 & \sum_{k=1}^{q}\left(P_{k} x_{M_{k}}^{1}\right) & \cdots & b_{m m} \sum_{k=1}^{q}\left(P_{k} x_{M_{k}}^{m}\right) \\
\sum_{k=1}^{q}\left(P_{k} x_{M_{k}}^{1}\right) & \sum_{k=1}^{q}\left[P_{k}\left(x_{M_{k}}^{1}\right)^{2}\right] & \cdots & \sum_{k=1}^{q}\left(P_{k} x_{M_{k}}^{m} x_{M_{k}}^{1}\right) \\
\vdots & \vdots & \cdots & \vdots \\
\sum_{k=1}^{q}\left(P_{k} x_{M_{k}}^{m}\right) & \sum_{k=1}^{q}\left[P_{k} x_{M_{k}}^{1} x_{M_{k}}^{m}\right] & \cdots & \sum_{k=1}^{q}\left[P_{k}\left(x_{M_{k}}^{m}\right)^{2}\right.
\end{array}\right], H=\left[\begin{array}{c}
a_{m} \\
b_{m 1} \\
\vdots \\
b_{m m}
\end{array}\right] \text {. }
$$

Obtain the parameter vector of predicting model.

$H=G^{-1} F$

$a_{m},{ }_{b m j}(j=1,2,3 \ldots m)$ into predicting formula(5) can obtain predicting value.

\section{The Analysis and Forecasting of Busy Traffic History Data}

Through data collection of daily busy traffic, obtained 350 busy traffic data, the data points $\left\{x_{1}, x_{2}, . ., x_{300}\right\}$ as a sample of forecasting model, $\left\{x_{301}, x_{302}, \ldots, x_{350}\right\}$ as test samples, the proposed approach is adopted to improve the step prediction research.

Analyzing of Chaos Characteristic. Select the autocorrelation function to calculate =2, though Cao's method to find $m=16$, and according to type (1), the 270 of $16 d$ phase sequence, the phase space to reconstruct the busy traffic expressed as follows.

$$
\left(\begin{array}{c}
X_{1} \\
X_{2} \\
\vdots \\
X_{270}
\end{array}\right)^{T}=\left(\begin{array}{cccc}
x_{1} & x_{3} & \cdots & x_{31} \\
x_{2} & x_{4} & \cdots & x_{32} \\
\vdots & \vdots & \cdots & \vdots \\
x_{270} & x_{272} & \cdots & x_{300}
\end{array}\right)^{T}
$$

Using trajectory algorithm to calculate $\max =0.046601>0$, which shows busy traffic has chaos characteristics. $1 /=21.459$ shows busy traffic is predictable, maximum forecast time is 21 days. 
Busy Traffic Prediction. Step projections for the latter 50 points. Prediction steps are as follows. Step 1, determine the center of prediction and forecasting phase points (Tab.1).

Table 1 Prediction center points and prediction phase points

\begin{tabular}{|c|c|c|}
\hline Prediction point & Prediction center point & Prediction phase point \\
\hline 301 & $X_{270}=\left[x_{270}, x_{272}, \ldots x_{300}\right]^{\mathrm{T}}$ & $X_{271}=\left[x_{271}, x_{273}, \ldots x_{301}\right]^{\mathrm{T}}$ \\
\hline 302 & $X_{271}=\left[x_{271}, x_{273}, \ldots x_{301}\right]^{\mathrm{T}}$ & $X_{272}=\left[x_{272}, x_{274}, \ldots x_{302}\right]^{\mathrm{T}}$ \\
\hline$\vdots$ & $\vdots$ & $\vdots$ \\
\hline
\end{tabular}

In Table 1, the $m$ th point of prediction phase points is the prediction value of prediction point.

Step 2, according to type (6) (8) calculate Euclidean distance and Angle cosine of $X_{i}$ and $X_{M}, y_{i}$ and $z_{i}$. Finally, calculate $c_{i}$, select $c_{i}$ as the largest of the $q$ value of the phase point as the reference neighborhood $X_{M k}$; determine the prediction center reference neighborhood;

Step 3, type (10) to calculate the k reference neighborhood point weights of $P_{k}$, calculate the forecast model parameter by type (13), and plug type (5) to get the predicted point prediction.

Step 4, repeat the above process, until the last data points is predicted.

Prediction value of and Angle cosine and observed data are shown in Tab.2.

Table 2 Observed data comparison with prediction value of this paper and angle cosin

\begin{tabular}{|c|c|c|c|c|c|c|c|}
\hline $\begin{array}{c}\text { data } \\
\text { point }\end{array}$ & $\begin{array}{c}\text { observed } \\
\text { data }\end{array}$ & $\begin{array}{c}\text { correlation } \\
\text { function }\end{array}$ & $\begin{array}{c}\text { angle } \\
\text { cosin }\end{array}$ & $\begin{array}{c}\text { data } \\
\text { point }\end{array}$ & $\begin{array}{c}\text { observed } \\
\text { data }\end{array}$ & $\begin{array}{c}\text { correlation } \\
\text { function }\end{array}$ & $\begin{array}{c}\text { angle } \\
\text { cosin }\end{array}$ \\
\hline 301 & 2.374 & 2.3485 & 2.2152 & 326 & 3.201 & 3.1155 & 3.4084 \\
\hline 302 & 2.763 & 2.6556 & 2.9289 & 327 & 2.293 & 2.1712 & 2.1351 \\
\hline 303 & 3.393 & 3.3094 & 3.2629 & 328 & 3.449 & 3.3878 & 3.3291 \\
\hline 304 & 2.971 & 2.8341 & 2.7929 & 329 & 3.149 & 3.237 & 2.9562 \\
\hline 305 & 2.511 & 2.488 & 2.6152 & 330 & 4.799 & 4.7024 & 4.9558 \\
\hline 306 & 3.376 & 3.5276 & 3.1967 & 331 & 4.159 & 4.0904 & 4.2652 \\
\hline 307 & 3.932 & 4.0216 & 4.1394 & 332 & 3.899 & 3.8315 & 4.0128 \\
\hline 308 & 3.216 & 3.1486 & 3.3178 & 333 & 3.003 & 2.8992 & 2.8799 \\
\hline 309 & 3.808 & 3.7158 & 3.7072 & 334 & 3.5 & 3.3919 & 3.3052 \\
\hline 310 & 3.402 & 3.2938 & 3.5668 & 335 & 3.496 & 3.3997 & 3.3256 \\
\hline 311 & 2.722 & 2.6323 & 2.989 & 336 & 3.141 & 3.0409 & 2.9481 \\
\hline 312 & 4.618 & 4.7783 & 4.4253 & 337 & 2.485 & 2.5815 & 2.2986 \\
\hline 313 & 4.094 & 4.2303 & 3.9204 & 338 & 2.431 & 2.3801 & 2.3075 \\
\hline 314 & 2.644 & 2.6736 & 2.4723 & 339 & 3.289 & 3.3599 & 3.1316 \\
\hline 315 & 2.746 & 2.8835 & 2.9369 & 340 & 3.103 & 3.201 & 2.9476 \\
\hline 316 & 3.968 & 4.0655 & 3.7985 & 341 & 4.362 & 4.4026 & 4.2273 \\
\hline 317 & 2.831 & 2.6955 & 2.6827 & 342 & 2.915 & 3.0063 & 3.0685 \\
\hline 318 & 3.9 & 3.9887 & 3.6981 & 343 & 4.05 & 4.0958 & 4.1657 \\
\hline 319 & 3.681 & 3.7301 & 3.4839 & 344 & 2.586 & 2.6915 & 2.4144 \\
\hline 320 & 3.965 & 3.85 & 3.8326 & 345 & 2.829 & 2.7317 & 2.9547 \\
\hline 321 & 4.361 & 4.4223 & 4.5274 & 346 & 3.064 & 2.9964 & 3.2716 \\
\hline 322 & 3.066 & 2.9418 & 2.9172 & 347 & 3.112 & 2.9845 & 3.3538 \\
\hline 323 & 2.46 & 2.3593 & 2.5851 & 348 & 5.012 & 4.9504 & 4.8366 \\
\hline 324 & 2.015 & 1.9035 & 2.1694 & 349 & 2.333 & 2.4497 & 2.5056 \\
\hline 325 & 3.3 & 3.2201 & 3.4256 & 350 & 3.435 & 3.3339 & 3.2216 \\
\hline
\end{tabular}

Prediction Evaluation. The absolute error of prediction is the difference of observed data and predicted value $(P=1,2, \ldots, 50)$, The paper adopts RMSE[10] to detect the effect of prediction.

$$
R M S E=\sqrt{\frac{1}{P} \sum_{p=1}^{P}\left(x_{N+p}-\hat{x}_{N+p}\right)^{2}}
$$

The comparison of RMSE is shown as Table 3. 
It can be seen by Table 3 that RMSE of correlation is less than Angle cosine, which shows that compared Angle cosine to measure phase point with correlation, the reference neighborhood and correlation of the prediction center correlation is stronger, obtained a good prediction effect.

Table 3 High and low settings of predictor variables

\begin{tabular}{|l|l|l|}
\hline error & correlation function & angle cosine \\
\hline RMSE & 0.00967 & 0.1666 \\
\hline
\end{tabular}

\section{Conclusions}

The paper reconstructed phase space of busy traffic and calculated $\quad \max =0.046601$ shows that busy traffic has both of chaos characteristics and predictability. Its prediction model is adding-weight one-rank local model;

In order to overcome the shortage of the phase correlation that adding-weighted one-rank local prediction model using Euclidean distance or Angle cosine measure separately, the article considers both of influence on phase correlation putting forward correlation function, a new adding-weighted one-rank local prediction algorithm is deduced. And forecast busy traffic. The result shows that the root mean square prediction error is 0.0967 , which shows that the correlation function is more suitable to describe the correlation between the phase points. The method is effective to improve the prediction precision of the busy traffic, and provides an effective way for the prediction of non-linear time series.

\section{References}

[1] MOU Ying, WANG Jun-feng, XIE Chuan-liu, etal. Forecast on traffic of large call center [J].Computer Engineering and Design, 2010,31(21):4686-4689,4719.

[2] ZENG Jun.Modeling and Simulation on Communication Traffic Forecasting Model, Computer Simulation, 2012, 29(2):116-119

[3] PENG Yu, WANG Jian-min, PENG Xi-yuan.Researches on Time Series Prediction with Echo State Networks [J].ACTA ELECTRONICA SINICA, 2010,38(2A):148-154.

[4] Han Min. Prediction Theory and Method of Chaotic Time Series [M].Beijing: China Water Power Press, 2007.

[5] LÜ Jin-hu, ZHANG Suo-chun. Application of adding-weight one-rank local-region method in electric power system short-term load forecast. Control Theory and Applications, 2002, 19(5):767-770.

[6] YUE Yi-Hong, HAN Wen-xiu,ZHANG Wei-bo.Local adding-weight linear regression forecasting method of chaotic series based on degree of incidence [J].Proceedings of the CSEE, 2004, 24(11):17-20.

[7] LEI Shao-Lan, SUN Cai-Xin, ZHOU Quan, etal. The Research on Short-term Load Forecasting Method Based on Improving Adding-weight One-rank Local Forecasting Model[J].Electrical Measurement \& Instrumentation, 2006,(5):5-8.

[8] WANG Huan, HE Yi-gang, GAO Kun. A Chaotic Local Power Forecasting Algorithm in Polar Coordinate System [J].Power System Technology, 2010,34(7):138-142.

[9] SUN Jialong, GUO Jinyun, GUO Shuyan. Analysis of Chaotic Properties and Prediction of Ionospheric Total Electron Content Based on Cosine of the Angle [J]. Bulletin of Surveying and Mapping, 2013, (5):8-11.

[10] Haiyan Wang,Lu-shan:Theory of Nonlinear Time Series Analysis and its Application(scinece Publications, China 2006),p.112-113. 